\title{
Esterilidad primaria de origen miomatoso curada por miomectomía
}

\author{
Dr. HÉCTOR ENRRIQUe BERNAL
}

Profescr Agregado de Ginecología. Adjunto del Departamento de Ginecología y Esterilidad del Hospital de San José.

En comunicaciones anteriores (1) (2) hemos tenido oportunidad de referirnos a los grandes factores ginecológicos como causa eficiente de infertilidad; entre ellos el mioma uterino (3) ocupa un lugar de importancia ya que si es verdad que numerosas miomatosas son capaces de llevar un embarazo a feliz término, en cambio hay otras en quienes la gestación fracasa precozmente, y por último un número quizá mayor para quienes la fecundación resulta prácticamente imposible.

No vamos a detenernos aquí sobre las distintas teorías que se han invocado para explicar la relación mioma-intertilidad; desde la clásica tesis de Seitz, quien definió el origen hormonal del tumor, la mayoría de los autores ha querido ver en los dos procesos manifestaciones diferentes de una sola causa, cual es la perturbación en el funcionamiento del ovario. Autores modernos, como Miller-Ludovitch y Dontas, de la Universidad de Michigan (4) por ejemplo, se refieren a la "alta incidencia de ciclos anovulatorios que permiten un prolongado estímulo estrogénico, sin interferencia de la progesterona y sobre pequeños nidos de células musculares inmaduras presentes en el miometrio".

Afortunadamente parece que no siempre el proceso es tan complicado, ya que hay casos en los cuales no queda duda de que el mioma es el único causante de la infertilidad; su papel entonces es de orden casi exclusivamente mecánico, ya sea por deformación de la cavidad uterina, por compresión o desplazamiento de la porción intersticial de las trompas, por edema, atrofia o ulceración del endometrio, etc. La conclusión lógica es que la cirugía conservadora, al suprimir el obstáculo, debe tener en estos casos un éxito indiscutible y precisamente a uno de ellos queremos referirnos en esta comunicación. 
Historia número 1756/53-1717.-I. B. S. Departamento de Ginecología. Sección de Esterilidad. Hospital de San José.

Se trata de una paciente de treinta años, de profesión hogar, natural de Gachetá, procedente de Bogotá y quien en febrero de 1953 consulta por esterilidad primaria con evolución de diez años, pues aun cuando tan sólo contrajo matrimonio hace cuatro, ha llevado vida sexual activa desde ia edad de veinte años. Menarquia a los trece años; ciclo desde un principio con tendencia a la hipermenorrea, últimamente con evolución aproximada de $\mathrm{V}$ a VII: 20-25-30. U. R. Se inicia el 5 de febrero al 28 o día de su cicio anterior.

Relaciones sexuales normales. Los demás antecedentes carecen de importancia.

Examen: Aspecto general: bueno. Tejido adiposo y distribución pilosa: normai. Aparato digestivo, respiratorio y circulatorio, en general normales. Pulso: 68' T. A. $14 \times 9$.

A la palpación abdominal se aprecia en el hipogastrio una masa del tamaño de una cabeza fetal de consistencia elástica dura ,de forma redondeada, bastante móvil en todo sentido e indolora.

Examen Ginecológico.- “Genitales externos": N. "Vagina" estrecha. "Utero" Cuello: de nulípara normal. Cuerpo: muy difícil de individualizar, parece estar englobado en la masa abdominal antes mencionada, la cual llena totalmente el fondo de Saco anterior y en parte los laterales y es irregular.

"Espéculo" Cuello y vagina de aspecto normal.

"Histerometría" 10 centímetros.

Tacto rectal: Confirma los datos anteriores; parametrios libres.

Diagnóstico.-Fibromioma uterino.

Se ordenan los exámenes de rutina, cuyos resultados son normales, salvo la presencia de ascárides que se tratan con Hexilresorcinol.

El mismo día (280 del ciclo), en las primeras horas de ia menstruación, se toma biopsia de endometrio cuyo resultado revela: endometrio de tipo secretor.

Con el fin de estudiar las posibiiidades de cirugía conservadora del fibromioma se practica Histerosalpingografía (Número 2953, julio 21 de 1953) que muestra (Figura 1) deformación de Ja cavidad uterina, y en la cual "a pesar de haber eievado la pre- 


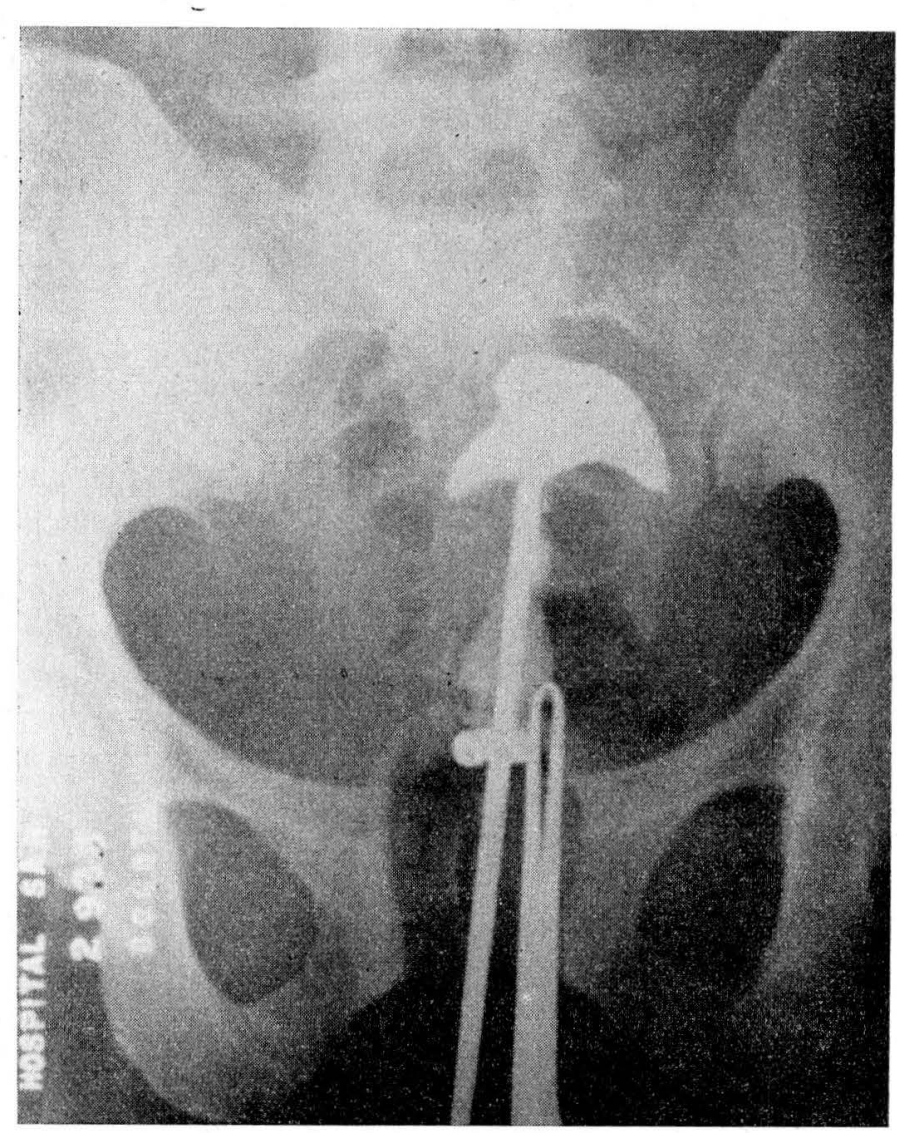

Figura 1

sión no se obtuvo paso a las trompas". Sin embargo, "en el control practicado a las 24 horas queda la impresión de que una parte del medio de contraste penetró a la cavidad peritoneal".

Ante esta posibilidad de permeabilidad tubárica se practica persuflación (agosto 19/53), en cuya gráfica se aprecia paso del gas a presiones entre 130 y 170 milimetros de $\mathrm{Hg}$. El peristaltismo aunque perezoso también exista.

La permeabilidad tubárica era pues indudable, por lo cual se decidió practicar ciruga conservadora.

El 15 de octubre de 1953 la paciente ingresa al servicio del profesor Brigard, en donde el 24 del mismo mes, previo neumo- 


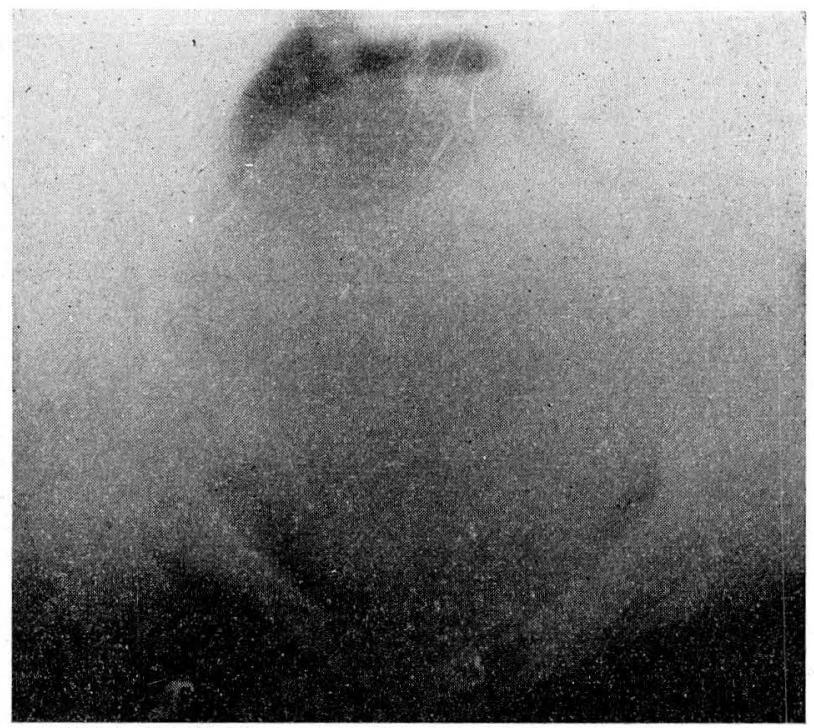

Figura 2

peritoneo, se practican ginecografías que muestran (figuras 2 y 3) la cavidad pélvica "ocupada por una masa de contorno regular que llena ia casi totalidad del espacio y que parece corresponder a un fibroma uterino. Una de las placas tomadas con mayor penetración (Figura 2) muestra otra imagen también circular, de diferente densidad, superpuesta a la opacidad primero descrita", y que el radiólogo interpreta como un nódulo miomatoso situado en plano diferente.

En estas condicions y previos los cuidados de rigor se interviene el 14 de noviembre de 1953.

Anestesia Raquídea. 2 horas.

Cirujanos: Héctor E. Bernal y Francisco Pardo V.

Descripción de la operación: Laparatomía media infraumbilical. Se encuentra matriz aumentada de tamaño y deformada por dos grandes nódulos miomatosos que lienan gran parte de 13 cavidad pśvica y localizados hacia el fondo uterino y pared anterior; hay tambión otros nódulos más pequeños e incluso dos pequeños miomas impiantados en cada uno de los ligamentos reciondos. El ovario derecho presenta degeneración escleroquistica.

Fracticada la hemostasis preventiva se procede a la enucleación de los varios nódulos miomatosos en cuya disección se lle- 


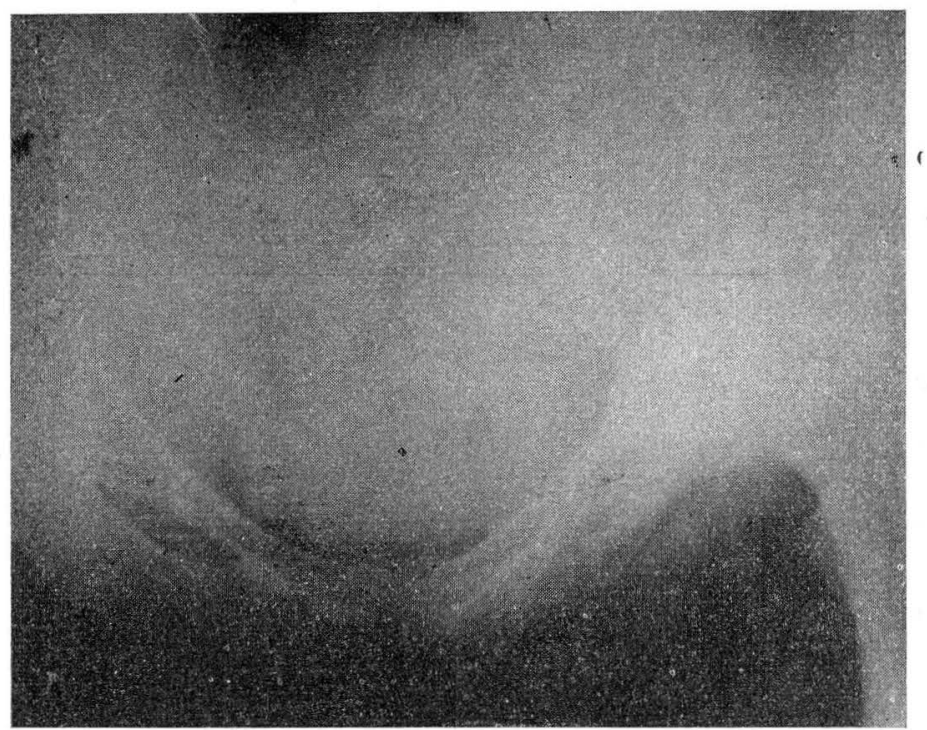

Figura 3

ga por delante hasta la propia cavidad uterina. Especialísimo cuidado se toma en el afrontamiento de los distintos planos musculares y se termina con resección cuneiforme del ovario derecho y apendicectomía. Cierre de la pared por planos.

Post-operatorio.--Perfectamente normal, lo que permitió dar de alta a la paciente al noveno día (23 de noviembre de 1953).

Al estudio anatomopatológico la masa mayor lobulada mide $10 \times 9 \times 7$ centímetros y los "cortes histológicos de los tumores muestran la estructura de fibromiomas".

Evolución.-Enero 7 de 1954. Al examen se aprecia la matriz de tamaño normal. Ha tenido dos menstruaciones con duración aproximada de 3 a 4 días. Para comprobar el resultado de la intervención se ordenó histerosalpingrafía con ginecografía que no se pudo practicar, pues la paciente se pierde de vista.

Noviembre 18 de 1954 . Ven a con ciclo de III: 28 normal y tuvo su última regla del 4 al 7 de septiembre de 1954; desde entonces en amenorrea por lo cual consulta. Al examen senos pigmentados y con secreción serosa. Cuello blando. Matriz uniformemente aumentada de tamaño y blanda, parece corresponder a. un embarazo de dos meses, de lo cual se da cuenta a la pacien- 
te advirtiéndole que debe continuar en observación en la consuita prenatal.

Sin embargo nuevamente se pierde de vista y es tan solo por intermedio de las enfermeras visitadoras como meses más tarde volvemos a establecer contacto para saber que el embarazo prosigue su curso y que es atendida en el I. C. S. S.

Debemos a la gentileza del doctor Belisario Calderón el habernos permitido consultar la correspondiente historia clínica de la cínica "Primero de Mayo" para continuar nuestra observación.

La paciente se inscribió en la consulta prenatal del I. C. S. S. ei 12 de enero de 1955 con diagnóstico de "Primigestante"; acusaba pequeñas hemorragias probablemente sin importancia por cuanto no hay observación especial; al examen clínico pulso 84, T. A. $15 \times 8$ altura uterina 11, auscultación y movimientos negativos; el resto del examen normal. En la segunda consulta (febrero 9) y posteriores los ruidos y movimientos fetales se hicieron aparentes y en estas condiciones normales continuó hasta el 3 de mayo de 1955, cuando por presentar cefalea, edemas, epigastralgia, y T. A. de $20 \times 10$ se ordenó hospitalización inmediata con el diagnóstico de "Toxemia" (preeclampsia grave).

Ingresa a la "Clínica Primero de Mayo" el 4 de mayo de 1955 con embarazo al $8^{\circ}$ mes en presentación O. I. A. encajada, T. A. de $190 \times 110$ y edemas. Al examen de orina huellas de albúmina y escasos hematies. Depuración ureica $70,5 \%$. Cuadro rojo: anemia de regular intensidad. Los otros exámenes en general normales.

Sometida a dieta aclorurada y tratamiento hipotensor, diurético sedante y antianémico evoluciona satisfactoriamente hasta el 29 de mayo de 1955 cuando inicia trabajo previa inducción con 5 unidades de pitocin en dextrosa al $5 \%$ que se suspende en virtud del antecedente quirúrgico. Finalmente, después de 12 horas, expulsa un feto vivo de sexo masculino con peso de 1.900 gramos y talla de 45 centímetros. El estado de la madre al salir es bueno con T. A. de $138 \times 70$ y en cuanto al niño pasa a la sección de prematuros en donde, sometido a los cuidados de rigor, progresa satisfactoriamente hasta aicanzar en julio 17, fecha de su salida, un peso de 2.500 gramos.

Posteriormente al practicar examen ginecoiógico a la madre hemos encontrado una matriz que involucionó perfectamen- 
te y en la que no se aprecian núdulos miomatosos. En cuanto al niño se encuentra en las condiciones normales propias de su edad.

\section{Comentarios}

El caso que acabamos de presentar constituye una feliz demostración de los beneficios de ia cirugía conservadora en el tratamiento del mioma uterino, con restitución completa de la función. Seguramente existen muchos semejantes entre nosotros que sería interesante conocer en benficio de la bibiografía nacional, y aunque ya desde 1941 el profesor Arturo Aparicio presentaba el primer caso de miomectomía seguida de una primera gestación, en paciente de veinticinco años (5), es poco lo que después se haya publicado en nuestro medio.

Revisando literatura de fuera vemos cómo Munnell y Martin (6), en un estudio sobre 236 miomectomias practicadas en el "Sloan Hospital for Women de New York", encuentran que 13 entre 23 pacientes con esterilidad primaria quedaron embarazadas y 11. llevaron su embarazo a feliz término hasta por un total de 18 embarazos. La mayor de dichas pacientes tenía cuarenta y dos años en el momento de la miomectomía.

Alfonso Alvarez (7) encuentra que sobre un total de 24 miomectomias practicadas a mujeres estériles el 30,4\% quedaron embarazadas en el curso de los dos primeros años. Borras en su comunicación al "Segundo Congreso Latinoamericano de Obstetricia y Ginecologia" dice: "Lo interesante es que de estas 284 enfermas 69 presentaron embarazos después de la operación, y de esas 69 enfermas 29 eran estériles, algunas con varios años de matrimonio antes de ser intervenidas". (8)

La insidencia de embarazo después de miomectomía se eleva según Davids, del "Mount Sinai Hospital" de New York, hasta el $50 \%$ (9).

Los resultados de la miomectomía en pacientes con aborto habitual, aunque no estadísticamente significativos, sugieren que la operación puede estar indicada en estos casos particularmente si el mioma es intramural; en el trabajo de Munnell 5 entre 9 pacientes que padec an de esta anomalía lograron llevar su embarazo a feliz término. Aunque no propiamente el tema de esta reseña, no está por demás recordar que según Davids sobre 35 pacientes a quienes se practicó miomectomía durante el embarazo 
el $65,7 \%$ llegaron a término. Casos semejantes han sido reportados entre nosotros por Correa (10), Bernal (3), Ofman (12) y especialmente Echeverry (13) quien presenta 5 casos con 4 éxitos.

La mayor:a de las pacientes que llevan su primer embarazo a término después de la operación tienen partos normales por vía vaginal; sin embargo an la estadística de Munnell hubo un alto porcentaje de sección cesárea $(17,6 \%)$. En dos casos la sección estaba indicada por infección de la cicatriz uterina en el post-operatorio (en uno de allos se produjo ruptura silenciosa del útero que se descubrió a la sección electiva). Algunos autores consideran como indicación de la cesárea las incisiones de miomectomía profundas y muy amplias; es interesante anotar sin embargo cómo $\epsilon \in \mathrm{n}$ algunas pacientes en quienes la incisión penetró a la cavidad uterina y que posteriormente fueron sometidas a cesáreas, no se ancontró endometrio en la cicatriz de miomectomía.

La ruptura uterina después de la miomectomía es rara comparada con su frecuencia después de la sección cesárea: la razón es porque el proceso de cicatrización se hace generalmente en circunstancias óptimas, como son una matriz no gestante y que no se contrae, la ausencia de infección frecuente en la sección cesárea y las suturas más pequeñas y menos numerosas. En la estaclística de Munnell, ya lo dijimos, tan solo hubo un caso de ruptura silenciosa en un útero cuya cicatriz se infectó en el postoperatorio, y lo mismo señala Borras, cuyo único caso se presentó en una enferma operada anteriormente para un fibroma ítsmico y embarazo, del cual abortó en el post-operatorio, y que seis años después hizo nuevo embarazo que terminó con un cuadro abdominal agudo por ruptura uterina, gran hemorragia e intenso shock. En cambio Alvarez no señala ningún caso de ruptura, de donde concluye que no hay razón alguna para impedir el embarazo durante el primer año del post-operatorio. En el caso que motiva esta comunicación no está por demás recordar que el parto fue inducido inicialmente con $5 \mathrm{U}$ de pitocín.

No quisiéramos terminar sin antes preguntarnos si existe o podría existir alguna relación entre la afección miomatosa y la intervención practicada a la paciente, y el estado de pre-eclampsia grave que presentó durante su embarazo. Evidentemente no parece existir ninguna relación de causa a efecto, ni tampoco lo confirma el estudio de las estadísticas antes mencionadas, en donde no se presenta ningún caso. Sin embargo no deja de ser interesante recordar la teoría de Sophian (Plaistow, England), quien 
considera la resistencia del miometrio a la extensión como causa de pre-eclampsia.

En su último trabajo (11) presenta datos experimentales y clínico-patológicos en apoyo de su concepto. Según él "la casi completa identidad de signos entre la nefritis aguda y la toxemia pre-eclámptica indica una base renai para esta última. "La preeclampsia ocurre en mujeres cuyo útero resiste en forma excesiva las tensiones normales o anormales propias del embarazo. En experimentos con animaies la extensión o estiramiento del útero produce por reflejo una alteración en la distribución de la corriente sangu'nea del riñón caracterizada por constricción de la arteria renal y espasmo progresivo de las arterias intralobulares y sus ramas glomerulares aferentes, desde la cápsula hacia adentro, en tanto que las porciones proximaies de las mismas arterias con sus ramas basales o "juxtamedullary glomeruli", escapan a :a constricción: de donde resulta "isquemia córtico-glomerular". La retención de agua y sal puede ocurrir, independientemente del exceso en la reabsorción tubular, como consecuencia del espasmo de las arteriolas glomerulares aferentes, mecanismo que también explicaría la hipertensión a albuminuria".

Dejamos al buen juicio del lector juzgar sobre la posible excesiva resistencia que un útero miomectomizado, pero cuya estructura celular completa es imposible de modificar, pueda ofrecer a la extensión determinada por el embarazo.

\section{BIBLIOGRAFIA}

1. BERNAL HECTOR E.- "Cómo conducir el interrogatorio en la presunta mujer esteril". "Revista Colombiana de Obstetricia y Ginecolcgía". Volumen VI. Número 2, noviembre-diciembre de 1954.

2. BERNAL HECTOR E.- "El factor ginecológico en el estudio de la esterilidad". Memorias de la Primera Convención Nacional de Obstetricia y Ginecología ("Revista Colombiana de Obstetricia y Ginecología". Volumen V. Número 5. 1954).

3. BERNAL HECTOR E.- "Niomatosis y Embarazo-Cirugía conservadora". "Repertorio de Medicina y Cirugía". Vol. V. Número 2. Enero-febrero de 1950.

4. MILLER-LUDOVICH-DONTAS.- "Problem o Uterine Fibroid" Am. J. Obst-Gynec. 66: 374, october. 1953. 
APARICIO JARAMILLO GUILLERN'O.- "Tratamiento Quirúrgico de los micmas uterinos-Miomectomía y Micmetrestomía”. Tesis de grado. Bogotá. 1941.

6. MUNNELL-MARTIN.- "Abdominal Myomectomy, Advantages and Disaduantages". Am. J. Obst. Gynec. 63: 317-325. February. 1952.

7. ALVAREZ BRAVO ALFONSO.- "Estudio de Esterilidad". Universidad Nacional de México. 4: 145-156. Julio 1953.

8. BORRAS PABLO E.- "Cirugía conservadora en Ginecología". "La Semana Médica”. Buenos Aires. Tomo 106. Número 12. Marzo. 1955.

9. DAVIDS ARTHUR N.--"Myomectomy Surgical Technic and Results in Series of 1.150 cases". Am. J. Obst-Gynec. 63: 292-604. March. 1952.

10. CORREA HUMBERTO.-“Cirugía conservadora en Ginecología". "Re. pertorio de Medicina y Cirugía”. Volumen IV. Número 5. Agosto de 1949.

11. SOPHIAN JOHN.-"Myometrial resistance to Strech cause of Preeclampsia”. J. Obst Gynec. Brist-Emp. 62: 37-47. February. 1955.

12. OFMAN G. JOSE.- "Comentarios sobre un caso de Fibromioma y Embarazo". "Revista Colombiana de Obstetricia y Ginecología". Volumen V. Número 3. Diciembre 1953 y enero 1954.

13. ECHEVERRY V. HERNANDO.- "Cinco casos de miomectomía durante el embarazo" "Revista Colombiana de Obstetricia y Ginecología". Volumen V. Número 4. Marzo, abril 1954. 